\title{
A GDOP-Weighted Intersection Method for Ray-Trace Based Target Localization using AOA Measurements in Quasi-Specular Environment
}

\author{
Fanzeng Kong ${ }^{a^{*}}$, Nae Zheng ${ }^{b}$, Song Chen ${ }^{c}$,Guojun Chen ${ }^{d}$ \\ Zhengzhou Institute of Information Science and Technology , China

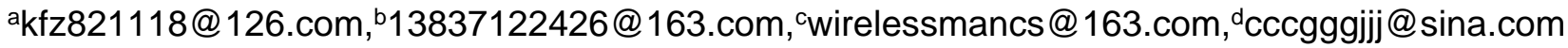

\begin{abstract}
Keywords: Localization; Angle of Arrival (AOA); Ray Tracing; Geometric Dilution of Precision (GDOP)
\end{abstract}

\begin{abstract}
Whereas environment modeling of current ray tracing algorithm used for locating is too ideal and the positioning accuracy is not high because AOA based weight intersection method fails to consider singularity bearing line intersection, this paper sets up AOA localization model on quasi-specular condition and proposes a GDOP-weighted intersection method (GWIAOA-RT) for ray-trace based target localization using AOA measurements. The method firstly utilizes idea of cluster to complete tracing propagation paths of multipath signal, gains virtual building surface after revised according to tracing result, constructs image base station, translates non-line-of-sight(NLOS) path into line-of-sight(LOS) path; then utilizes AOA measurements on each path and gets all intersections of bearing line; further utilizes circular error probable (CEP) to determine effective intersections, gives corresponding weight according to Geometric Dilution of Precision (GDOP) of effective intersections, and weighted merge them to estimate the position of target. The method utilizes multipath signal to eliminate the impact of NLOS noise on positioning accuracy as well as consideration of position error of image base station in GDOP calculation. It has strong robustness. Simulation result shows that compared with existing methods, GWIAOA-RT method has higher positioning accuracy and better adaptation in different application scenarios.
\end{abstract}

\section{Introduction}

Location based service(LBS) provides great convenience for people's life. It has become an important component in modern life. Precise location information is the basis of LBS, so localization technique is always the research focus. Among many localization techniques, localization technique based on ray tracing $[1,2]$ can effectively solve problems affecting positioning effect seriously like multipath propagation, hearability [3] and NLOS noise, etc. faced by Range-based localization method and thus is paid extensive attention.

Localization technique based on ray tracing utilizes the various measurements of signal to realize the tracing of signal propagation path, constructs virtual base station with LOS path and applies various range-based localization method to target position calculating. There are some literatures to study ray tracing used for positioning. Ray tracing methods of signal based on multi-sensors were proposed in $[4,5]$. Ray tracing methods based on single sensor were proposed in [6,7]. The above signal propagation environmental modeling of algorithms is too ideal and affects the practicability of algorithm. Ref.[8] thinks the fluctuation surface of water changes with time is not pure plain. When signal reflects under water, reflex angle is determined jointly by the tangent plane at reflection point by fluctuation surface and incident ray. In such situation, reflex angle is deviated from that on pure plain condition and such environment modeling is closer to reality.

Typical range-based localization method mainly includes Time of Arrival (TOA) based method[9], Time Difference of Arrival (TDOA) based method[10] and Angle of Arrival (AOA) based method[11,12], etc. Of them, AOA based method is an important research branch. This paper mainly studies AOA based localization method and Cramér-Rao lower bound based on ray tracing.

Related scholars have made many researches on localization method based on AOA. Stansfield[13] proposed a weighted least square method. In [14], Pseudo Linear-squares (PLS) was proposed and 
gives closed form solution of target position. Such two methods don't need initial estimation of target position and are easy to be realized, but both of them have biased estimate. Methods given by [15], [16-18]are maximum likelihood estimation. They realizes asymptotic unbiased estimation under Gaussian assumed conditions. The method performance approaches Cramer-Rao Low Bound (CRLB). Such methods are iterative methods, and need initialized position which is closer to real position. If initialized position is unreasonable, it may lead to that method is converged by local optimal solution or cannot converge [19].

In [20-22], the intersections of bearing line(IBL) between sensor and target are utilized for weighted summation to estimate position of target. In [22] complex AOA(CAOA) method was proposed. This method considers the mean value of all bearing line intersections as estimated position of target. Localization method based on sensitivity analysis was proposed in [23], generate corresponding weighted value according to first order biased derivatives of closed form solution of bearing line intersection and merge them. Reference [20] [21] suggested bearing line intersection based localization method on the basis of positioning error analysis. Weighted intersection AOA (WIAOA) localization method proposed in [20] utilizes the distance among sensors, AOA measurements and their standard deviation to construct the weighted value of bearing line intersection. CRLB-Weighted Intersection AOA (CWIAOA) location method proposed in [21] utilizes CRLB of bearing line intersection to get weighted value of intersection. The positioning accuracy of WIAOA and CWIAOA method is superior to that of PLS method, but it fails to consider the following condition ,i.e. some intersections have greater weighted value, but the deviation from actual position of target is greater than that of intersection with small weighted value. Such intersection used for target position estimation may affect the accuracy of estimation seriously. Such intersection can be called singularity intersection of bearing line (SIBL).

This paper firstly establishes AOA based localization model on quasi-specular condition according to microcell environment where base station (BS) and mobile station (MS) locate. Secondly, it gives a GDOP-weighted intersection method (GWIAOA-RT) for ray-trace based target localization using AOA measurements. Such method utilizes cluster based ray tracing method to trace propagation path of multipath signal and gives position formula of image base station (I-BS) position according to mirror principle and revised virtual building surface. It utilizes I-BSs and their AOA measurements constructed by ray tracing to get all IBLs, considers the remaining IBLs as effective intersection and weighted merge them, according to circular error probable to get rid of SIBLs. Based on this, it estimates target position. The method can make full use of multipath propagation of signal to realize single station location. It amends the position of I-BSs on quasi-specular condition and considers the position error of I-BSs in the weighted value calculation to make method have better robustness for positioning accuracy and application environment. At last, simulation result shows that compared with existing methods, this method have higher positioning accuracy and better adaptation in different application scenarios.

\section{AOA Location Model in Microcell}

The model of this method includes two parts: signal propagation environment model and localization model.

\subsection{Signal Propagation Environment Model}

It needs to construct environment model of wireless signal propagation directing at geographical information around the target to trace signal path. Such model is made up of geometric model and morphological model. Geometric model can be gained through field measurement or GIS system. The method given in this paper is mainly used for microcell, so we can assume that the ground of target area is horizontal flat ground. In order to simplify the model, and establish it in 2D plane, the geometric model of building is polygon formed by its projection on the ground. The side of polygon is the surface of building, the ray will be reflected on the surface, and the ith surface can be expressed with the following formula

$$
F_{i}: a_{i} x+b_{i} y+c_{i}=0 \quad x_{i, \min } \leq x \leq x_{i, \max }, y_{i, \min } \leq y \leq y_{i, \max }
$$


where $x_{i, \min }=\min \left(x_{i, 1}, x_{i, 2}\right), x_{i, \max }=\max \left(x_{i, 1}, x_{i, 2}\right), y_{i, \min }=\min \left(y_{i, 1}, y_{i, 2}\right), y_{i, \max }=\max \left(y_{i, 1}, y_{i, 2}\right)$, $\left(x_{i, 1}, y_{i, 1}\right)$ and $\left(x_{i, 2}, y_{i, 2}\right)$ are the coordinates of terminals of the $\mathrm{i}^{\text {th }}$ side that is the projection of the $\mathrm{i}^{\text {th }}$ surface in the horizontal plane. The intersection of two sides is the intersected arris of building surfaces, and the ray will be diffracted when reaching arris.

Morphological model mainly includes parameters affecting signal propagation path. It's impossible that geometric model depicts all details on the surface of building. If assuming the surface of building is pure mirror, such assumption is too ideal, failing to consider the convexes or concaves existed on the surface of building, or the impact on signal propagation path by some uncertain factors. Referring to [8], we need comprehensively consider various factors on the surface of building that may cause impact on signal propagation in order to make signal propagation model in microcell closer to reality. This paper thinks that when signal is reflected on the surface of building, reflected ray will have certain derivation compare with the pure specular reflection, thus a small deflection angle $\theta_{F}$ will be added, as is shown in Fig.1. Under this circumstance, the surface of building is called quasi-mirror and signal propagation environment model based on quasi-mirror is called quasi-mirror signal propagation model. Since building is fixed, when signal is reflected on the surface of building, the deflection angle will be invariable for certain incidence point, but for the whole surface of building, deflection angle can be assumed to follow the zero-mean normal distribution with the variance as $\delta_{F}^{2}$. Meanwhile, the value of $\delta_{F}$ shall not be too big, which is assumed to be the range of $0^{\circ}$ to $3^{\circ}$.

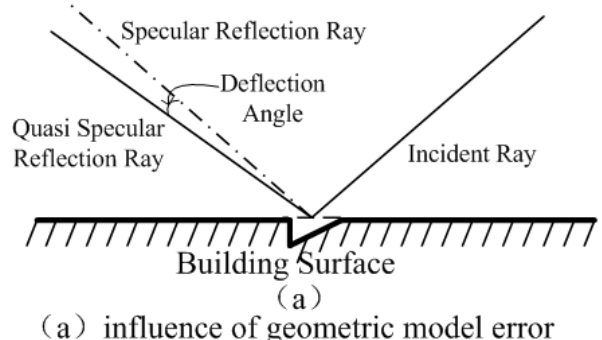

(a) influence of geometric model error on the reflected ray

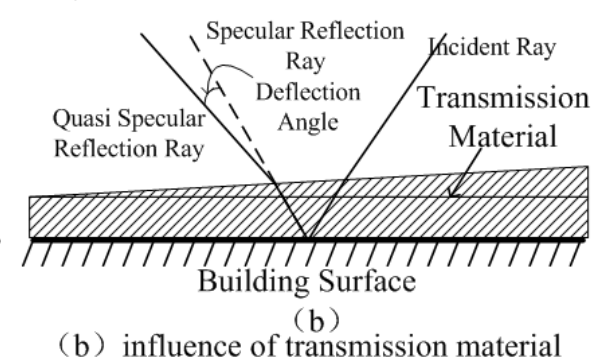

(b) influence of transmission material on reflection ray

Figure 1. reflection schematic diagram on the surface of building

During propagation process, only direct or reflection happens for signal, thus the formed signal propagation path is called position orientating path (POP). Such signal is called position orientating signal (POS). For POP, I-BS can be determined by utilizing mirror reflection principle, thus NLOS path can be translated into LOS path to I-BS. $\mathrm{N}$ times of reflection happens in propagation process of POS, thus such signal is called $\mathrm{N}$ order POS, the propagation path is called $\mathrm{N}$ order POP. In order to describe it conveniently, LOS path is also called 1 order POP.

\subsection{Localization Model}

Ray tracing based AOA method translates the angles of arrival of multipath signals measured by BS into angles of arrival of I-BSs to realize the solution of target position. BS receives signal through array antenna and utilizes beam forming, MUSIC algorithm or ESPRIT algorithm to realize AOA estimation.

Assuming that there are $N$ POPs between BS and MS, $N$ I-BSs can be determined, the known position and real position of the $\mathrm{i}^{\text {th }} \mathrm{I}$-BS are expressed with $\tilde{\mathbf{X}}_{i}=\left[\tilde{x}_{i}, \tilde{y}_{i}\right]^{T}$ and $\mathbf{X}_{i}=\left[x_{i}, y_{i}\right]^{T}$ respectively, the relation can be expressed with the following formula:

$$
\tilde{\mathbf{X}}_{i}=\mathbf{X}_{i}+\mathbf{N}_{i}, \mathbf{N}_{i}=\left[n_{x i}, n_{y i}\right]
$$

In the formula, $n_{x i}$ and $n_{y i}$ are the error of known position of I-BS at the direction of $\mathrm{X}$ and $\mathrm{Y}$ axis.

$\tilde{\theta}_{i}$ indicates AOA measurement on the $\mathrm{i}^{\text {th }} \mathrm{I}-\mathrm{BS}, \theta_{i}$ indicates AOA real value on the ith I-BS, $n_{i}$ indicates error of AOA measured value on the $\mathrm{i}^{\text {th }} \mathrm{I}-\mathrm{BS}$, it complies with normal distribution with zero mean and standard deviation $\sigma_{i}$. The position of target is unknown, and its real position is expressed 
with $p_{T}=\left[x_{T}, y_{T}\right]^{T}$. The estimation result of target position by method is expressed with $\hat{p}_{T}=\left[\hat{x}_{T}, \hat{y}_{T}\right]^{T}$. AOA measurements can be expressed as:

$$
\begin{aligned}
& \tilde{\Theta}=\Theta+\mathbf{N}_{\theta} \\
& \theta_{i}=f_{i}\left(p_{T}\right)=\arctan \left(\frac{y_{T}-y_{i}}{x_{T}-x_{i}}\right)
\end{aligned}
$$

where, $\tilde{\Theta}=\left[\tilde{\theta}_{1}, \cdots, \tilde{\theta}_{N}\right]^{T}, \Theta=\left[\theta_{1}, \cdots, \theta_{N}\right]^{T}, \mathbf{N}_{\theta}=\left[n_{1}, \cdots, n_{N}\right]^{T}$..

\section{GDOP-Weighted Intersections for Ray-Tracing-Based Target Localization Using AOA Measurements}

\subsection{Clustering analysis based ray tracing method (CART)}

To realize tracing of POP, we need to find out POS from multipath signals and complete tracing of propagation path. However, in the process of ray tracing, if we don't consider AOA estimation error and deflection angle caused owing to reflection on the surface of building, the estimation of propagation path will be greatly influenced and the tracing path will deviate from real path seriously, i.e. the result of ray tracing deviates the passing building or incidences on the error building surface.

In order to solve the above problems, during the process of POP ray tracing, we can introduce several paths from BS and reflection point respectively according to every signal received, get multiple possible propagation paths (feasible path) through continuous iteration, and select optimal path among all feasible paths as the result of tracing.

Let's take Fig. 2 for example. For a signal, we can take BS as starting point, and introduce $M$ paths in the range of $\left[\theta_{A O A}-\delta_{A O A}, \theta_{A O A}+\delta_{A O A}\right]$, where $\theta_{A O A}$ is estimation of AOA by BS, and $\delta_{A O A}$ is standard deviation of estimation. The angle of every path is expressed as:

$$
\begin{aligned}
& \theta_{A O A m}=\theta_{A O A}-\delta_{A O A}+(m-1) \Delta \theta_{A O A}, m=1, \ldots, M \\
& \Delta \theta_{A O A}=2 \delta_{A O A} /(M-1)
\end{aligned}
$$

Carry out collision test for multipath led from BS in microcell based on geometric model, find out reflecting surface and calculate the position of reflection point. According to specular reflection principle, calculate reflective angle $\theta_{R A 1}, \ldots, \theta_{R A M}$ of every path at the position of reflection point, and then lead to $N$ reflective paths at each reflection point in the range of $\left(\theta_{R A m}-\delta_{F}, \theta_{R A m}+\delta_{F}\right)$. Of them, $\delta_{F}$ is standard deviation of deflection angle on the surface of building, and the angle of every reflective path is:

$$
\begin{aligned}
& \theta_{R A m, n}=\theta_{R A m}-\delta_{F}+(n-1) \Delta \theta_{F} \quad m=1, \ldots, M, n=1, \ldots, N \\
& \Delta \theta_{F}=2 \delta_{F} / N-1
\end{aligned}
$$

Carry out collision test for every reflective path again, find the next reflecting surface and repeat this process until the ending conditions of tracing is realized.

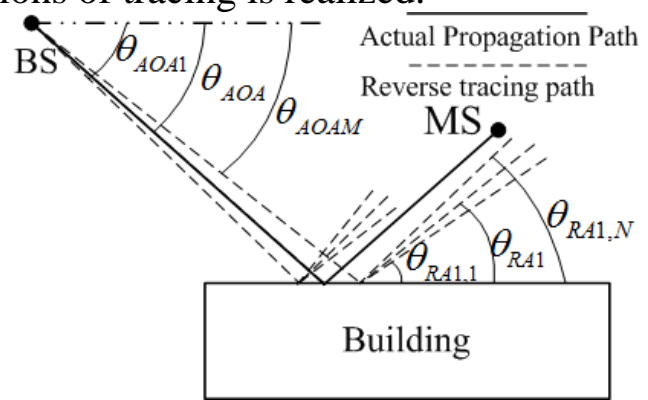

Figure 2. Position oriented path inverse ray tracing schematic diagram

In [1-3] ,the declining energy of signal is too great after reflected for three times or above, so BS cannot receive such signal. Therefore, if the length of tracing path meets $L=c \tau$, where $L$ is length of tracing path, $c$ is velocity of light, $\tau$ is estimation of time delay of signal, then the tracing of this path will be terminated. When the length of tracing path has experienced reflection for three times before 
meeting the above formula, the tracing of such path will be considered as a failure and then terminate the tracing.

Look toward MS from BS, the terminal points in feasible paths of multipath signal got in the above method consistent with real propagation path gather around actual position of MS. Set the terminal of the $\mathrm{i}^{\text {th }}$ signal on the $\mathrm{j}^{\text {th }}$ feasible path as $\mathbf{X}_{i, j}^{p}$, utilize systematical cluster analysis thought for the terminals $\mathbf{X}_{i, j}^{p}$ of all feasible paths, and find out paths that terminal of path gathers around target. Select the classification with highest degree of gathering at terminal points of feasible paths as optimal classification $G_{0}$ with the gravity center as $\bar{X}_{0}$.

If a signal gets the terminal point of feasible path according to the method, and it belongs to $G_{0}$ we get after cluster analysis, thus this signal is POS. If multiple terminal points of feasible paths of a POS belong to $G_{0}$, then the feasible path through which the terminal point is nearest to gravity center $\bar{X}_{0}$ is deemed as the optimal tracing path of the signal propagation path.

In conclusion, the procedures of clustering analysis based ray tracing method is summed as the following: 1) according to TOA and AOA measurements of multipath signal received by BS, get feasible paths of each path; 2) make cluster analysis of terminal points of all feasible paths and determine optimal classification; 3) according to optimal classification, determine POS and the optimal propagation path.

\subsection{Position of Image Base Station}

The known position and real position of BS are expressed with $\tilde{\mathbf{X}}_{0}=\left[\tilde{x}_{0}, \tilde{y}_{0}\right]^{T}$ and $\mathbf{X}_{0}=\left[x_{0}, y_{0}\right]^{T}$ respectively, $n_{x 0}$ and $n_{y 0}$ are the error of known position of BS at the direction of $\mathrm{X}$ axis and $\mathrm{Y}$ axis. $n_{x 0}$ and $n_{y 0}$ comply with normal distribution with zero-mean, then the standard deviation of $n_{x 0}$ is $\sigma_{x 0}$ and the standard deviation of $n_{y 0}$ is $\sigma_{y 0}$ respectively, and they are not related to each other. Their relation can be expressed with the following formula.

$$
\tilde{\mathbf{X}}_{0}=\mathbf{X}_{0}+\mathbf{N}_{0}, \mathbf{N}_{0}=\left[n_{x 0}, n_{y 0}\right]
$$

There are $N$ POPs between BS and MS, so $N$ I-BSs can be determined. $\tilde{\mathbf{X}}_{i}=\left[\tilde{x}_{i}, \tilde{y}_{i}\right]^{T}$ and $\mathbf{X}_{i}=\left[x_{i}, y_{i}\right]^{T}$ shows respectively the known position and real position of the $\mathrm{i}^{\text {th }} \mathrm{I}$-BS, $\tilde{\mathbf{X}}_{i}$ is got according to $\tilde{\mathbf{X}}_{0}$ and the corresponding POP.

When carrying out environment modeling, this paper assumes that the surface of building as quasi-speculum, so when ray is reflected on the surface of building, incident angle may not be equal to reflex angle. In this case, to avoid increasing additional error in position estimation of MS, we can revise the reflected surface of building and construct a virtual construction surface. As is shown in Fig.3, the signal is reflected on the surface $F_{i}$ of building, rotate angle $\beta$ with the center of reflection point $\mathbf{X}^{P}$, and make incident angle $\theta$ equal to reflex angle $\alpha$. According to analytic geometry and formula (1), we can get the formula of virtual building surface, which is expressed as:

$$
\begin{aligned}
& F_{i} \stackrel{x^{p}, \beta}{\longrightarrow} \tilde{F}_{i} \\
& \begin{cases}F_{i}: a_{i} x+b_{i} y+c_{i}=0 & x_{i, \text { min }} \leq x \leq x_{i, \text { max }}, y_{i, \text { min }} \leq y \leq y_{i, \text { max }} \\
\tilde{F}_{i}: a_{i}^{\prime} x+b_{i}^{\prime} y+c_{i}^{\prime}=0 & x_{i, \text { min }}^{\prime} \leq x \leq x_{i, \text { max }}^{\prime}, y_{i, \text { min }}^{\prime} \leq y \leq y_{i, \text { max }}^{\prime}\end{cases}
\end{aligned}
$$

where $\tilde{F}_{i}$ is surface got by $F_{i}$ rotate angle $\beta$ with the center of reflection point $\mathbf{X}^{P}$.

If the $\mathrm{i}^{\text {th }}$ POS reflects on $N_{i}$ building surfaces $\left(F_{i_{n}}, n=1, \cdots, N_{i}\right)$, according to real propagation path and (10),we can get $\tilde{F}_{i_{n}}$ by revising $F_{i_{n}}$. Then according to image theory, we get $N_{i}$ order I-BS of BS corresponding to the path, the position of the I-BS can be expressed as:

$$
\left\{\begin{array}{l}
\tilde{\mathbf{X}}_{i}=\mathbf{A}_{i} \tilde{\mathbf{X}}_{0}+\mathbf{B}_{i} \\
\mathbf{X}_{i}=\mathbf{A}_{i} \mathbf{X}_{0}+\mathbf{B}_{i}
\end{array}\right.
$$


where $\mathbf{A}_{i}=\prod_{n=N_{i}}^{1} \mathbf{A}_{i, n}=\mathbf{A}_{i, N_{i}} \mathbf{A}_{i, N_{i}-1} \cdots \mathbf{A}_{i, 1}, \mathbf{B}_{i}=\sum_{n=1}^{N_{i}} \mathbf{A}_{i}^{n} \mathbf{B}_{i, n}, \mathbf{A}_{i}^{m}= \begin{cases}\prod_{n=N_{i}}^{m+1} \mathbf{A}_{i, n} & , m<N_{i} \\ \mathbf{I} & , m=N_{i}\end{cases}$

$\mathbf{A}_{i, n}=\left[\begin{array}{cc}\frac{b_{i_{n}}^{\prime 2}-a_{i_{n}}^{\prime 2}}{a_{i_{n}}^{2}+b_{i_{n}}^{2}} & \frac{-2 a_{i_{n}}^{\prime} b_{i_{n}}^{\prime}}{a_{i_{n}}^{2}+b_{i_{n}}^{2}} \\ \frac{-2 a_{i_{n}}^{\prime} b_{i_{n}}^{\prime}}{a_{i_{n}}^{\prime 2}+b_{i_{n}}^{\prime 2}} & \frac{a_{i_{n}}^{\prime 2}-b_{i_{n}}^{\prime 2}}{a_{i_{n}}^{\prime 2}+b_{i_{n}}^{\prime 2}}\end{array}\right], \mathbf{B}_{i, n}=\left[\begin{array}{c}\frac{-2 a_{i_{n}}^{\prime} c_{i_{n}}^{\prime}}{a_{i_{n}}^{\prime 2}+b_{i_{n}}^{\prime 2}} \\ \frac{-2 b_{i_{n}}^{\prime} c_{i_{n}}^{\prime}}{a_{i_{n}}^{\prime 2}+b_{i_{n}}^{\prime 2}}\end{array}\right]$.

In addition, if the $\mathrm{i}^{\text {th }}$ POP is a LOS path, then $\mathbf{A}_{i}=\mathbf{I}$ and $\quad \mathbf{B}_{i}=\mathbf{0}$. Make $\tilde{\mathbf{X}}_{s}=\left[\tilde{\mathbf{X}}_{1}^{T}, \cdots, \tilde{\mathbf{X}}_{N}^{T}\right]^{T}, \quad \mathbf{X}_{s}=\left[\mathbf{X}_{1}^{T}, \cdots, \mathbf{X}_{N}^{T}\right]^{T}, \quad \mathbf{A}=\left[\mathbf{A}_{1}^{T}, \cdots, \mathbf{A}_{N}^{T}\right]^{T}$, $\mathbf{B}=\left[\mathbf{B}_{1}^{T}, \cdots, \mathbf{B}_{N}^{T}\right]^{T}$, and we can get the following formula according to(10) and (11).

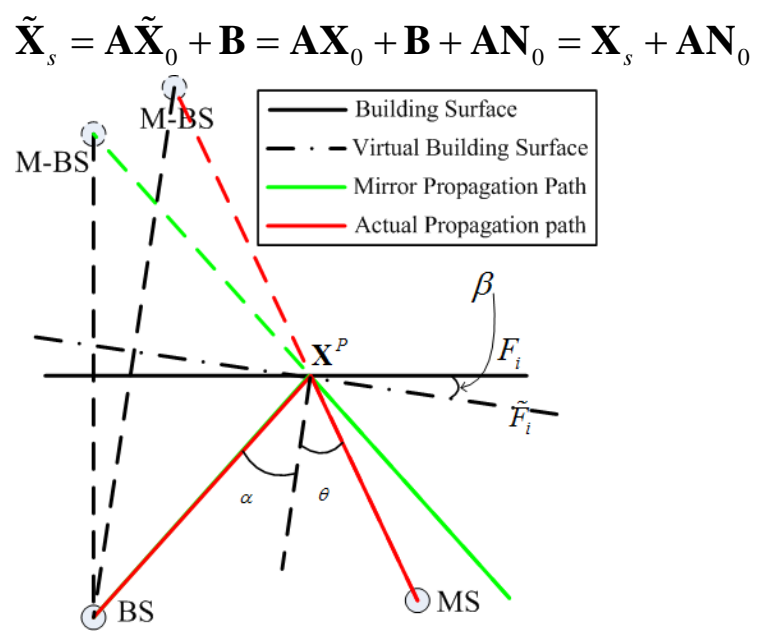

Figure 3. Image base station schematic diagram

\subsection{GDOP-Weighted Intersection Localization Method Based on AOA}

On the basis of that BS completes ray tracing of signal and gets I-BSs, we can estimate the MS position by AOA measurements at I-BSs. To simplify the problem, we assume the relationship between BS position and I-BSs position is accurate, namely $\mathbf{A}$ and $\mathbf{B}$ in formula (12) are accurate. In order to improve positioning accuracy of bearing line intersection based AOA method, we can get rid of SIBL. Thus, we can consider introducing circular error probable (CEP) to solve this problem. On 2D conditions, circular error probable is defined as the probability that positional result falls into circle area $\mathrm{C}$ with real position of MS as center. Circle area $\mathrm{C}$ is called error probability circle, its radius is defined as $n$ times of radius of GDOP [24] and determined by (13). Of them, $R_{C E P-P}$ indicates the radius of circle area $C$ into which the positioning result falls with probability $P$. According to [24], in (13), the relation between $P$ and $n$ can be determined by (14) and (15).

$$
\begin{aligned}
& R_{C E P-P}=n \cdot G D O P \\
& P=\int_{0}^{n} 2 \rho e^{-\rho^{2}} \mathrm{~d} \rho=1-\mathrm{e}^{-n^{2}} \\
& n=\sqrt{-\ln (1-P)}
\end{aligned}
$$

The center of circle area $\mathrm{C}$ is real position of MS, while the position of MS in localization is unknown. Here, substitute real position $\left(x_{T}, y_{T}\right)$ of MS with IBL $\hat{p}_{i, j}=\left[\hat{x}_{T}^{i, j}, \hat{y}_{T}^{i, j}\right]$, we can get the radius of error probability circle can be got from the following formula:

$$
R_{C E P-P}^{i, j} \approx R_{C E P-P} \mid x_{T}=\hat{x}_{T}^{i, j}, y_{T}=\hat{y}_{T}^{i, j}
$$

where $R_{C E P-P}^{i, j}$ indicates the radius of error probability circle at intersection of the $\mathrm{i}^{\text {th }}$ bearing line and $\mathrm{j}^{\text {th }}$ bearing line. There are $C_{N}^{2}$ intersections for $N$ bearing lines, if the distance $d_{i 1, j 1, i 2, j 2}$ between IBL $\hat{p}_{i_{1}, j_{1}}$ and $\hat{p}_{i_{2}, j_{2}}$ meets: 


$$
d_{i_{1}, j_{1}, i_{2}, j_{2}}<R_{C E P-P}^{i_{1}, j_{1}} \quad \text { or } \quad d_{i_{1}, j_{1}, i_{2}, j_{2}}<R_{C E P-P}^{i_{2}, j_{2}}
$$

thus IBL $\hat{p}_{i_{1}, j_{1}}$ and $\hat{p}_{i_{2}, j_{2}}$ are considered as effective intersections. Under the circumstance that probability is $P$, find out effective intersections and use effective intersection for position estimation of MS to reduce the impact of SIBL on positioning accuracy.

We will discuss GDOP calculation problem below. In consideration of error of I-BSs, according to (4), make differentiation for $\theta_{i}$ and get:

$$
\mathrm{d} \theta_{i}=\frac{\partial f_{i}(p)}{\partial x_{T}} \mathrm{~d} x_{T}+\frac{\partial f_{i}(p)}{\partial y_{T}} \mathrm{dy}_{T}+\frac{\partial f_{i}(p)}{\partial x_{i}} \mathrm{~d} x_{i}+\frac{\partial f_{i}(p)}{\partial y_{i}} \mathrm{dy}_{i}
$$

where $u=\frac{y_{T}-y_{i}}{x_{T}-x_{i}}, \frac{\partial f_{i}(p)}{\partial x_{T}}=-\frac{\partial f_{i}(p)}{\partial x_{i}}=\frac{-u}{1+u^{2}} \frac{1}{x_{T}-x_{i}}, \frac{\partial f_{i}(p)}{\partial y_{T}}=-\frac{\partial f_{i}(p)}{\partial y_{i}}=\frac{1}{1+u^{2}} \frac{1}{x_{T}-x_{i}}$.

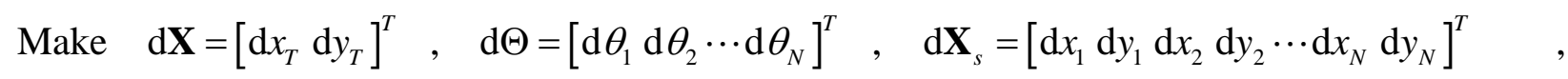

$\mathbf{C}_{1}^{x}=\left[\frac{\partial f_{1}(p)}{\partial x_{T}}, \cdots, \frac{\partial f_{N}(p)}{\partial x_{T}}\right]^{T}, \mathbf{C}_{1}^{y}=\left[\frac{\partial f_{1}(p)}{\partial y_{T}}, \cdots, \frac{\partial f_{N}(p)}{\partial y_{T}}\right]^{T}, \mathbf{C}_{2}^{i}=\left[\frac{\partial f_{i}(p)}{\partial x_{i}}, \frac{\partial f_{i}(p)}{\partial y_{i}}\right], \mathbf{C}_{1}=\left[\begin{array}{ll}\mathbf{C}_{1}^{x} & \mathbf{C}_{1}^{y}\end{array}\right]$, $\mathbf{C}_{2}=\operatorname{diag}\left(\mathbf{C}_{2}^{1}, \cdots, \mathbf{C}_{2}^{N}\right)$, Get

$$
\mathbf{C}_{1} \mathrm{~d} \mathbf{X}=\mathrm{d} \Theta-\mathbf{C}_{2} \mathrm{~d} \mathbf{X}_{s}
$$

By use of pseudo inverse, get position error estimation of target:

$$
\begin{gathered}
\mathrm{d} \hat{\mathbf{X}}=\mathbf{D}\left(\mathrm{d} \Theta-\mathbf{C}_{2} \mathrm{~d} \mathbf{X}_{s}\right) \\
\mathbf{P}_{\mathrm{d} \hat{\mathbf{X}}}=\mathrm{E}\left[\mathrm{d} \hat{\mathbf{X}} \mathrm{d} \hat{\mathbf{X}}^{T}\right]=\mathbf{D}\left\{\mathrm{E}\left[\mathrm{d} \Theta \mathrm{d} \Theta^{T}\right]+\mathbf{C}_{2} \mathrm{E}\left[\mathrm{d} \mathbf{X}_{s} \mathrm{~d} \mathbf{X}_{s}{ }^{T}\right] \mathbf{C}_{2}^{T}\right\} \mathbf{D}^{T}
\end{gathered}
$$

where $\mathbf{D}=\left(\mathbf{C}_{1}^{T} \mathbf{C}_{1}\right)^{-1} \mathbf{C}_{1}^{T}$. Assuming AOA measurement errors at I-BSs are unrelated to each other, then

$$
\mathrm{E}\left[\mathrm{d} \Theta \mathrm{d} \Theta^{T}\right]=\operatorname{diag}\left(\sigma_{1}^{2}, \sigma_{2}^{2}, \cdots, \sigma_{N}^{2}\right)
$$

According to (12), the position of each I-BS is determined by the position of BS and model of microcell jointly. Assuming various elements of position error of BS are not related to each other, then

$$
\mathrm{E}\left[\mathrm{d} \mathbf{X}_{s} \mathrm{~d} \mathbf{X}_{s}^{T}\right]=\mathbf{A}\left(\begin{array}{cc}
\sigma_{x 0}^{2} & 0 \\
0 & \sigma_{y 0}^{2}
\end{array}\right) \mathbf{A}^{T}
$$

Make $\mathbf{P}_{\mathrm{d} \hat{\mathrm{x}}}=\left[\begin{array}{cc}\sigma_{\hat{x}}^{2} & \sigma_{\hat{x} \hat{y}} \\ \sigma_{\hat{y} \hat{x}} & \sigma_{\hat{y}}^{2}\end{array}\right]$, then GDOP $=\sqrt{\sigma_{\hat{x}}^{2}+\sigma_{\hat{y}}^{2}}$.

Here, we still substitute real position of target with intersection of the $\mathrm{i}^{\text {th }}$ bearing line and $\mathrm{j}^{\text {th }}$ bearing line, and get

$$
\begin{aligned}
& \mathbf{P}_{\mathrm{d} \hat{\mathrm{x}}}^{i, j}=\mathbf{P}_{\mathrm{d} \hat{\mathrm{x}}} \mid x_{T}=\hat{x}_{T}^{i, j}, y_{T}=\hat{y}_{T}^{i, j} \\
& G D O P_{i, j}=G D O P \mid x_{T}=\hat{x}_{T}^{i, j}, y_{T}=\hat{y}_{T}^{i, j}
\end{aligned}
$$

Then the intersection of the ith bearing line and jth bearing line is $\hat{p}_{i, j}=\left[\hat{x}_{T}^{i, j}, \hat{y}_{T}^{i, j}\right]^{T}$, and the non-normalized weight on $\mathrm{X}$ axis and $\mathrm{Y}$ axis is:

$$
\tilde{\omega}_{i, j}^{x}=\left(\mathbf{P}_{\mathrm{d} \hat{\mathrm{X}}}^{i, j}(1,1)\right)^{-1}, \tilde{\omega}_{i, j}^{y}=\left(\mathbf{P}_{\mathrm{d} \hat{\mathrm{X}}}^{i, j}(2,2)\right)^{-1}
$$

The set formed by all effective IBLs is $\hat{\mathbf{P}}$, make

$$
\mathbb{I}\left(\hat{p}_{i, j}\right)= \begin{cases}1 & \hat{p}_{i, j} \in \hat{\mathbf{P}} \\ 0 & \hat{p}_{i, j} \notin \hat{\mathbf{P}}\end{cases}
$$

Normalize the weight of effective IBLs $\left(\hat{p}_{i, j} \in \hat{\mathbf{P}}\right)$

$$
\omega_{i, j}^{x}=\mathbb{I}\left(\hat{p}_{i, j}\right) \tilde{\omega}_{i, j}^{x}\left(\sum_{m=1}^{N-1} \sum_{n=m+1}^{N} \mathbb{I}\left(\hat{p}_{m, n}\right) \tilde{\omega}_{m, n}^{x}\right)^{-1}, \omega_{i, j}^{y}=\mathbb{I}\left(\hat{p}_{i, j}\right) \tilde{\omega}_{i, j}^{y}\left(\sum_{m=1}^{N-1} \sum_{n=m+1}^{N} \mathbb{I}\left(\hat{p}_{m, n}\right) \tilde{\omega}_{m, n}^{y}\right)^{-1}
$$


If IBL $\hat{p}_{i, j}$ doesn't belong to $\hat{\mathbf{P}}$, then

$$
\omega_{i, j}^{x}=0, \omega_{i, j}^{y}=0 \quad \hat{p}_{i, j} \notin \hat{\mathbf{P}}
$$

According to (28) and (29), weighted sum IBLs, the estimation of MS position is

$$
\left[\hat{x}_{T}, \hat{y}_{T}\right]^{T}=\left[\sum_{i=1}^{N-1} \sum_{j=i+1}^{N} \omega_{i, j}^{x} \hat{x}_{T}^{i, j}, \sum_{i=1}^{N-1} \sum_{j=i+1}^{N} \omega_{i, j}^{x} \hat{y}_{T}^{i, j}\right]^{T}
$$

To sum up, GWIAOA-RT method is proposed in this section mainly includes the following four steps: 1) get measurements of TOA and AOA of multipath signal;2) utilize CART to identify POSs in multipath signal and realize path tracing; 3) gain position of I-BSs and their AOA measurements; 4) realize the estimation of MS position. The specific steps of GWIAOA-RT method are as the following table.

Table 1. GWIAOA-RT Method

\begin{tabular}{l}
\hline \multicolumn{1}{|c|}{ GWIAOA-RT method steps } \\
\hline Initialization: base station $\tilde{\mathbf{X}}_{0}$, TOA and AOA measurements of multipath signal , circular error probable $P=0.99$ Method process: \\
STEP1: utilize CART method to determine POSs in multipath framing signal and realize tracing of their propagation paths; \\
STEP2:according to ray tracing result and geometric model of ray propagation environment, utilize (10-12) to gain position of I-BSs $\mathbf{X}_{s}$ and \\
their corresponding AOA measurements $\tilde{\Theta}=\left[\tilde{\theta}_{1}, \cdots, \tilde{\theta}_{N}\right]^{T} ;$ \\
STEP3: utilize GDOP-weighted intersection localization method based on AOA to realize estimation of target position; \\
STEP3-1:according to gained bearing line intersection $\hat{p}_{i, j}=\left[\hat{x}_{T}^{i, j}, \hat{y}_{T}^{i, j}\right] ;$ \\
STEP3-2: calculate $G D O P_{i, j}$ according to (25); \\
STEP3-3: calculate $R_{C E P-P}^{i, j}$ according to (16); \\
STEP3-4:calculate the distance between IBLs, and determine effective intersection set $\hat{\mathbf{P}}$ according to (17); \\
STEP3-5: calculate weight of each IBL according to (24-29); \\
STEP3-6: estimate position of MS according to (30).
\end{tabular}

\section{Cramer-Rao Low Bound}

Assuming in the microcell environment, there are N POPs between base station $\left(x_{0}, y_{0}\right)$ and target $\left(x_{T}, y_{T}\right)$ based on signal propagation model. According to (10-12), we can get $N$ I-BSs, $\mathbf{X}_{i}(i=1 \cdots N)$. According to [21], Fisher information matrix of MS is $\mathbf{J}=\sum_{i=1}^{N} \mathbf{M}_{i}$, where

$$
\mathbf{M}_{i}=\left[\begin{array}{cc}
\frac{\left(y_{T}-y_{i}\right)^{2}}{\sigma_{s}^{2}\left(\left(x_{T}-x_{i}\right)^{2}+\left(y_{T}-y_{i}\right)^{2}\right)^{2}} & \frac{-\left(y_{T}-y_{i}\right)\left(x_{T}-x_{i}\right)}{\sigma_{s}^{2}\left(\left(x_{T}-x_{i}\right)^{2}+\left(y_{T}-y_{i}\right)^{2}\right)^{2}} \\
\frac{-\left(y_{T}-y_{i}\right)\left(x_{T}-x_{i}\right)}{\sigma_{s}^{2}\left(\left(x_{T}-x_{i}\right)^{2}+\left(y_{T}-y_{i}\right)^{2}\right)^{2}} & \frac{\left(x_{T}-x_{i}\right)^{2}}{\sigma_{s}^{2}\left(\left(x_{T}-x_{i}\right)^{2}+\left(y_{T}-y_{i}\right)^{2}\right)^{2}}
\end{array}\right]
$$

Its CRLB matrix is $\mathbf{C}=\mathbf{J}^{-1}$, and then CRLB of method can be got from CRLB matrix:

$$
C R L B=\sqrt{\operatorname{Trace}(\mathbf{C})}
$$

\section{Simulation Result and Analysis}

To verify the performance of the method given in this paper, this section makes contrast of positioning performance of GWIAOA-RT method, CAOA method, WIAOA method, CWIAOA method and SA method in two typical scenarios. In each scenario, Monte-Carlo simulation is carried out for 1000 times, then we can collect statistics on Root Mean Square Error (RMSE) and Cumulative Distribution of position error of each method.

Fig. 4 gives building layout of microcell where BS and MS located, and propagation paths of POS on the condition of pure specular environment (PSE) and quasi specular environment (QSE). POP on the condition of quasi specular environment is established according to signal propagation model in Section II. 
Simulation will be carried out in two scenarios. In scenario 1, there are 1-order and 2-order POPs, and I-BSs are on both sides of MS. In scenario 2, there are only 1-order POPs, and all I-BSs are located on one side. In these two scenarios, we can analyze the impact of different I-BS distributions on positioning accuracy of method.

Scenario 1: there are four buildings in microcell, their projection coordinates at ground are known, and shown as Table 2 . The real coordinate of BS is $(0,45)$, and the real coordinate of MS is $(240,40)$. There are five 1-order and two 2-order POPs between BS and MS (as is shown in Table 3), namely seven I-BSs in total, as is shown in Fig.4. BS can get AOA measurements of the POPs. According to the relationship between BS position and I-BSs position, AOA measurement of each POP at BS converts the AOA measurement at corresponding at I-BSs. AOA measurement error at I-BSs complies with Gaussian distribution with zero mean, and standard deviation is $\sigma_{1}=\cdots=\sigma_{7}=\sigma_{\theta}$, which are unrelated to each other, while the value range of $\sigma_{\theta}$ is $\left[1^{\circ}, 5^{\circ}\right]$. BS has error $n_{x 0}$ and $n_{y 0}$ on $\mathrm{X}$ axis and $\mathrm{Y}$ axis at the known position, and it complies with normalized distribution with zero-mean and the standard deviation is $\sigma_{x 0}=\sigma_{y 0}=\sigma_{s}=1$, which are unrelated to each other.

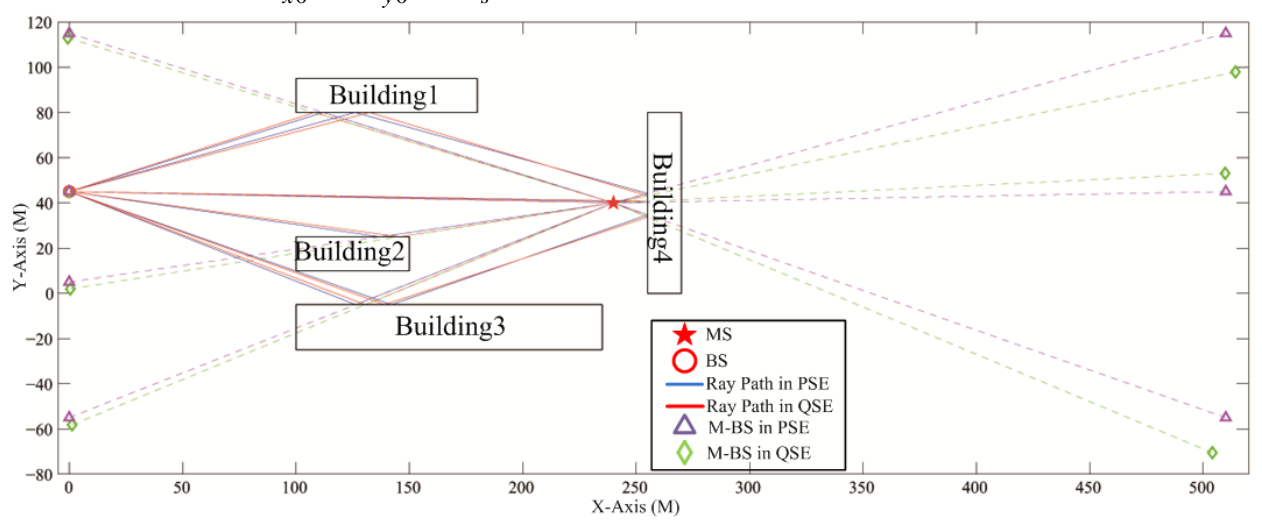

Figure 4. microcell

Table 2. Building Coordinate within Microcell Table 3. P-path Coordinate on Quasi-specular

\begin{tabular}{|c|c|c|c|c|c|c|c|c|}
\hline Building & $\mathrm{X} 1$ & $\mathrm{Y} 1$ & $\mathrm{X} 2$ & $\mathrm{Y} 2$ & $\mathrm{X} 3$ & $\mathrm{Y} 3$ & $\mathrm{X} 4$ & $\mathrm{Y} 4$ \\
\hline Building 1 & 100 & 80 & 100 & 95 & 180 & 95 & 180 & 80 \\
\hline Building 2 & 100 & 10 & 100 & 25 & 150 & 25 & 150 & 10 \\
\hline Building 3 & 100 & -25 & 100 & -5 & 235 & -5 & 235 & -25 \\
\hline Building 4 & 255 & 0 & 255 & 80 & 270 & 80 & 270 & 0 \\
\hline
\end{tabular}

Condition within Mmicrocell

\begin{tabular}{|c|c|c|c|c|c|c|c|c|}
\hline & $\mathrm{X} 1$ & $\mathrm{Y} 1$ & $\mathrm{X} 2$ & $\mathrm{Y} 2$ & $\mathrm{X} 3$ & $\mathrm{Y} 3$ & $\mathrm{X} 4$ & $\mathrm{Y} 4$ \\
\hline POP1 & 0 & 45 & 240 & 40 & & & & \\
\hline POP 2 & 0 & 45 & 108.1 & 80 & 240 & 40 & & \\
\hline POP 3 & 0 & 45 & 145.7 & 25 & 240 & 40 & & \\
\hline POP 4 & 0 & 45 & 130.6 & -5 & 240 & 40 & & \\
\hline POP 5 & 0 & 45 & 255 & 40.7 & 240 & 40 & & \\
\hline POP 6 & 0 & 45 & 132.5 & 80 & 255 & 43.2 & 240 & 40 \\
\hline POP 7 & 0 & 45 & 132.5 & 80 & 255 & 33.7 & 240 & 40 \\
\hline
\end{tabular}

Fig.5 is curve of RMSE of each method changing with $\sigma_{\theta}$ in scenario 1, and Fig.6 is accumulative distribution of each method in scenario $1\left(\sigma_{\theta}=5^{\circ}\right)$. From Fig.5, we can see, in scenario 1 , the performance of CAOA method is the worst, the overall performance of GWIAOA-RT method is superior to that of other methods, and the deviation degree of RMSE of CAOA and SA method is increased obviously with the increasing of $\sigma_{\theta}$. Because known position of BS used for MS position solution has error, RMSE of all methods has certain deviation compared with CRLB. From Fig.6, we can see, when $\sigma_{\theta}=5^{\circ}$, the positioning accuracy of GWIAOA-RT method is still higher than other methods.

Scenario 2: assuming there are Building1, 2 and 3 in microcell, and the projection coordinate at level is shown as Table 2 . The real coordinate of BS is $(0,45)$,and the real coordinate of MS is (240, 40). There are four 1-order POPs between BS and MS, namely four I-BSs in total, and all I-BSs are located on one side, as is shown in Fig.4. Assuming that the tracing result of CART method for all POPs is identical to real path, AOA measurement error at I-BSs complies with Gaussian distribution with zero-mean, and standard deviation is $\sigma_{1}=\cdots=\sigma_{4}=\sigma_{\theta}$, which are unrelated to each other, the value range of $\sigma_{\theta}$ is $\left[1^{\circ}, 5^{\circ}\right]$. BS has error $n_{x 0}$ and $n_{y 0}$ on $\mathrm{X}$ axis and $\mathrm{Y}$ axis at the known position, it 
complies with normalized distribution with zero-mean and the standard deviation is $\sigma_{x 0}=\sigma_{y 0}=\sigma_{s}=1$, which are unrelated to each other.

Fig.7 is curve of RMSE of each method changing with $\sigma_{\theta}$ in scenario 2, Fig.8 is accumulative distribution of each method in scenario $2\left(\sigma_{\theta}=5^{\circ}\right)$. From Fig.7, we can see, in scenario 2, the performance of CAOA method is the worst, the performance of SA method is superior to CAOA method. Because known position of BS has error, RMSEs of CAOA method and SA method deviate from CRLB, while the positioning performance of GWIAOA-RT, WIAOA and CWIAOA method is close when $\sigma_{\theta}$ is small, but with the increasing of $\sigma_{\theta}$ continuously, the performance of GWIAOA-RT will be superior to that of other methods. From Fig.8, we can see, when $\sigma_{\theta}=5^{\circ}$, the overall performance of GWIAOA-RT method is still superior to that of other methods.

Comparing scenario 1 with scenario 2, we can see the positioning performance of the above kinds of methods is better in scenario 1 than in scenario 2, that is to say, when I-BSs are distributed around target, the positioning performance of methods is better. Meanwhile, because the known position of BS has error, RMSE of the above five kinds of methods has certain deviation compared with CRLB. From Fig.5 and Fig.7, we can see, for GWIAOA-RT, WIAOA and CWIAOA method, the impact of $\sigma_{s}$ will be weakened relatively with the increasing of $\sigma_{\theta}$ in the case that $\sigma_{s}$ is fixed.

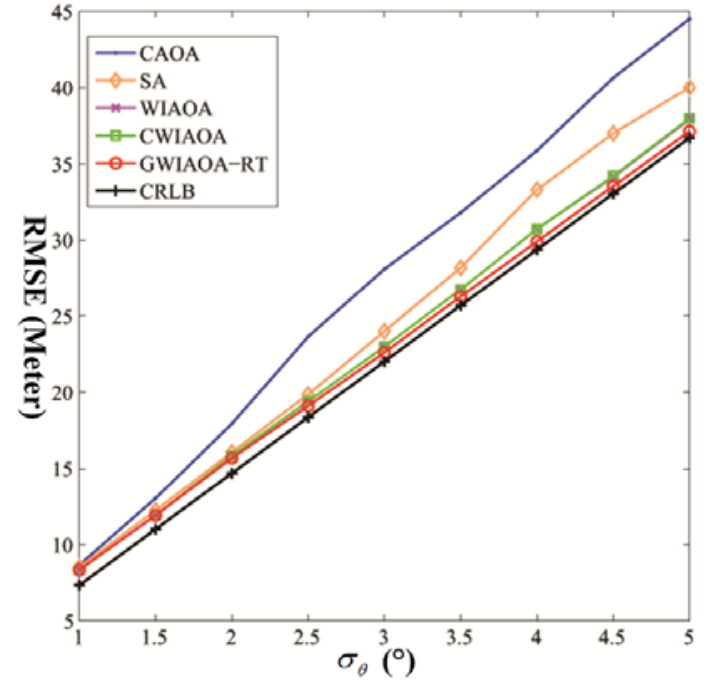

Figure 5. RMSE of methods in scene 1

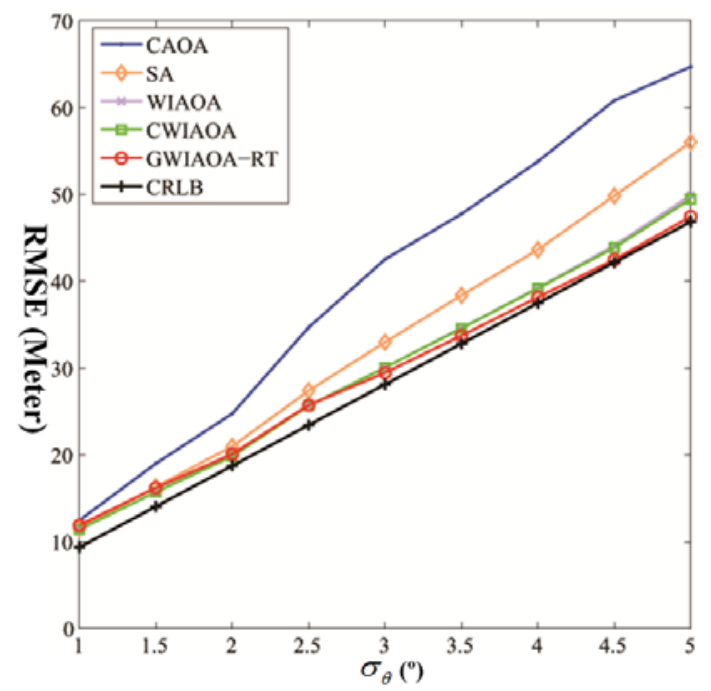

Figure 7. RMSE of various methods in scene 2

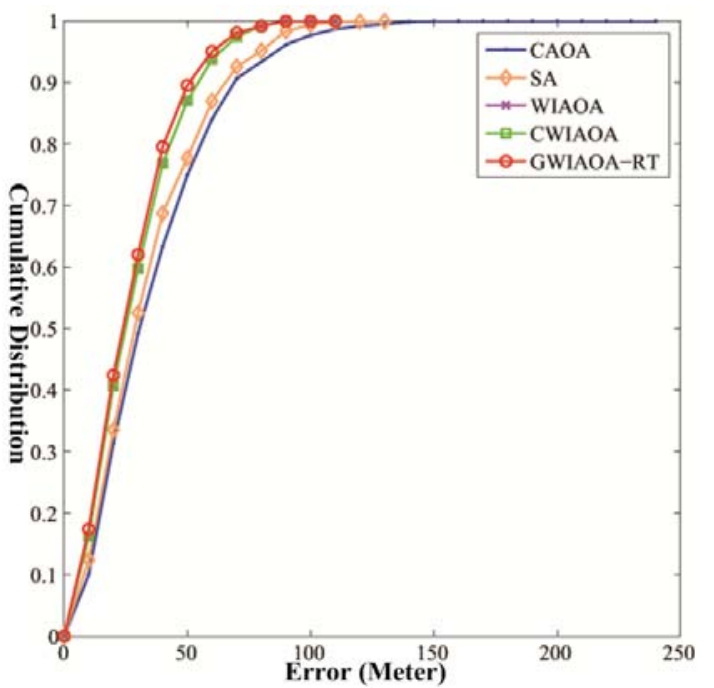

Figure 6. error accumulation distribution of

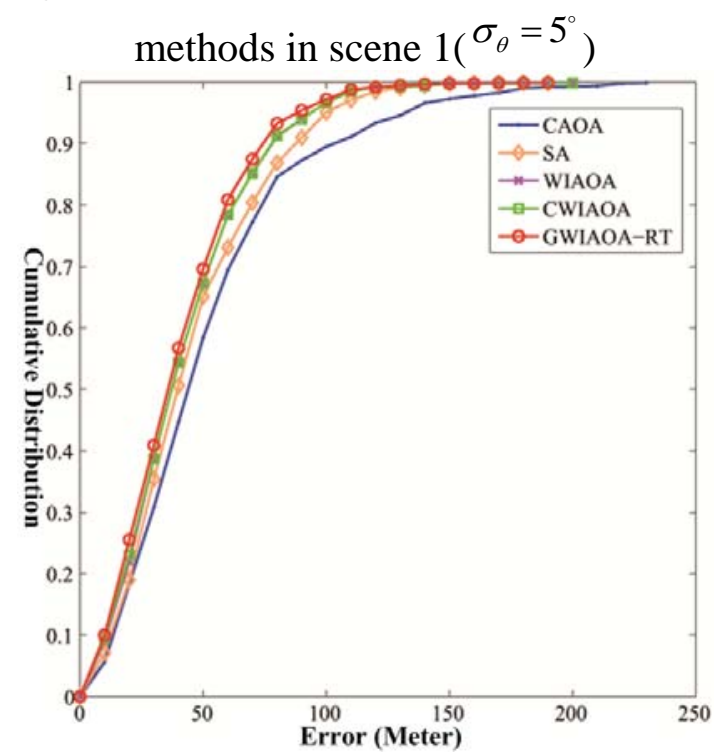

Figure 8. error accumulation distribution of method in scene $2\left({ }^{\sigma_{\theta}}=5^{\circ}\right)$ 
Simulation result shows that GWIAOA-RT method given in this paper in different simulation scenarios has better adaptability than other kinds of methods and the positioning accuracy of such method is superior to other methods in the whole.

\section{Conclusion}

This paper gives a GDOP-weighted intersection method for ray-trace based target localization using AOA measurements on quasi-specular condition. The core thought of the method is to utilize cluster analysis thought to realize ray tracing, construct multiple I-BSs at BS according to relative measurements of multipath signal, utilize circular error probable to determine effective intersection among all IBLs, give corresponding weight according to GDOP, merge them and get position estimation of MS. GWIAOA-RT method revises the building surface passed by POP, makes the calculation of I-BS position more accurate, and reduces the impact on solution precise of MS position. Meanwhile, the impact of position error of BS on result in GDOP calculation is considered, and the method is not an iterated method, i.e. it doesn't need initialized value. And MS position estimate of GWIAOA-RT method can be used as the initialized value of the other positioning method. Simulation result indicates, compared with WIAOA method, CWIAOA method SA method and CAOA method, GWIAOA-RT method has better adaptability and the overall positioning accuracy of the method is higher.

\section{Acknowledgment}

This work is supported by the National Natural Science Foundation of China (No.61401513), all support is gratefully acknowledged.

\section{References}

[1] X. P. Zhou, F. J. Tan, C. Y. Liu and P. Zou. 2013. An improved algorithm on field strength calculation in ray tracing technology, Chinese Journal of Radio Science, 28(4): 669-675.

[2] A. Schmitz, T. Rick, T. Karolski, T. Kuhlen and L. Kobbelt. 2011. Efficient Rasterization for Outdoor Radio Wave Propagation, IEEE Transactions on Visualization \& Computer Graphics, 17(2): 159-170.

[3] Z. W. Yuan. 2003. The Theories and Methods of the Mobile Terminals Positioning in Cellular Communieations Systems Based on Ray-Tracing, Changsha: Central South University.

[4] K. W. K. Lui and H. C. So. 2010. Range-based Source Localication with Pure Reflector in Presence of Multipath Propagation, Electronics Letters, 46(8):957-958.

[5] D. L. Liu, K. Liu, Y. Ma and J. Yu. 2014. Joint TOA and DOA Localization in Indoor Environment Using Virtual Station, IEEE Communications Letters, 18(8):1423-1426.

[6] P. Setlur, G. E. Smith, F. Ahmad and M.G. Amin. 2012. Target Localization with a Single Sensor via Multipath Exploitation, IEEE Transactions on Aerospace and Electronic Systems, 48(3):1996-2014.

[7] J. H. Wang. 2011. Research on Cellular Location Techniques Based on Information Fusion, Zhengzhou: PLA Information Engineering University.

[8] L. E. Emokpae, S. Dibenedetto, B. Potteiger and M. Younis. 2014. UREAL: Underwater Reflection-Enabled Acoustic-Based Localization, IEEE Sensors Journal, 14(11): 3915-3925.

[9] G. H. Zhu, D. Z. Feng, Y. Zhou and W. K. Nie. 2015. TOA localization algorithm using the linear-correction technique, Journal of Xidian University (Natural Science), 42(3):22-25.

[10] M . Compagnoni and R. Notari. 2014. TDOA--based localization in two dimensions: the bifurcation curve, Fundamenta Informaticae, 135: 199-210. 
[11] H. J. Shao, X. P. Zhang and Z. Wang. 2014. Efficient Closed-Form Algorithms for AOA Based Self-Localization of Sensor Nodes Using Auxiliary Variables, IEEE Transactions on Signal Processing, 62(10):2580-2594.

[12] S. C. K. Herath and P. N. Pathirana. 2013. Optimal Sensor Arrangements in Angle of Arrival (AoA) and Range Based Localization with Linear Sensor Arrays, Sensors, 13(9): 12277-12294.

[13] R. Stansfield. 1947. Statistical theory of d.f. fixing, Journal of the Institution of Electrical Engineers-Part IIIA: Radiocommunication, 94(15):762 - 770.

[14] A. Pages-Zamora, J. Vidal, and D. H. Brooks. 2002. Closed-form Solution for Positioning Based on Angle of Arrival Measurements, in Proceedings of the 13th IEEE International Symposium on Personal, Indoor and Mobile Radio Communications, 4:1522-1526.

[15] W. Foy. 1976. Position-location solutions by Taylor-series estimation, IEEE Transactions on Aerospace and Electronic Systems, 12(2):187-194.

[16] D. Torrieri. 1984. Statistical theory of passive location systems, IEEE Transactions on Aerospace and Electronic Systems, 20:183-198.

[17] Z. Berman. 1997. A reliable maximum likelihood algorithm for bearing-only target motion analysis, in Proceedings of the 36th IEEE Conference on Decision and Control, 5: 5012-5017.

[18] Z. Wang, J. A. Luo, and X. P. Zhang. 2012. A novel location-penalized maximum likelihood estimator for bearing-only target localization, IEEE Transactions on Signal Processing, 60:6166-6181.

[19] J.-P. Le Cadre and C. Jaetffret. 1999. On the convergence of iterative methods for bearings-only tracking, IEEE Transactions on Aerospace and Electronic Systems, 35:801-818.

[20] Q. Zhou and Z. S. Duan. 2014. Weighted intersections of bearing lines for AOA based localization, Information Fusion (FUSION) :1-8.

[21] Z. S. Duan and Q. Zhou. 2015. CRLB-weighted intersection method for target localization using AOA measurements, Computational Intelligence and Virtual Environments for Measurement Systems and Applications (CIVEMSA): 1-6.

[22] P. Brida, J. Machaj, J. Benikovsky, and J. Duha. 2010. A new complex angle of arrival location method for ad hoc networks, In Proceedings of the 7th Workshop on Positioning Navigation and Communication: 284-290.

[23] M. Soltanian, A. M. Pezeshk, A. Mahdavi, and M. Dallali. 2011. A new iterative position finding algorithm based on taylor series expansion, In Proceedings of the 19th Iranian Conference on Electrical Engineering: 1-4.

[24] K. Yu, I. Sharp and Y. J. Guo. 2009. Ground-Based Wireless Positioning, Wiley-IEEE Press, Chichester. 